\title{
Clinical impacts of a micropapillary pattern in lung adenocarcinoma: a review
}

This article was published in the following Dove Press journal:

OncoTargets and Therapy

31 December 2015

Number of times this article has been viewed

\section{Ying Cao \\ Li-Zhen Zhu \\ Meng-Jie Jiang \\ Ying Yuan}

Department of Medical Oncology, The Second Affiliated Hospital,

Zhejiang University School of

Medicine, Hangzhou, People's

Republic of China
Correspondence: Ying Yuan

Department of Medical Oncology,

The Second Affiliated Hospital, Zhejiang

University School of Medicine, 88 Jiefang

Road, Hangzhou 310009, People's

Republic of China

Tel +86 57l 87784795

Fax +86 57। 872I 4404

Email yuanying1999@zju.edu.cn

\begin{abstract}
Lung adenocarcinoma with a micropapillary pattern (MPPAC) has recently drawn increased attention among researchers. Micropapillary-predominant adenocarcinoma (MPA), which is defined by micropapillary pattern (MPP), is the primary histological pattern observed semiquantitatively in 5\% increments on resection specimens, and MPA was formally determined to be a new histological subtype according to the new multidisciplinary classification in 2011. According to published studies, MPPAC is most common in males and nonsmokers and is associated with lymphatic invasion, pleural invasion, and lymph node metastases. MPPAC often presents as part-solid and lobulated nodules in computed tomography scans. MPP tends to have a higher maximum standardized uptake value as determined by fluorodeoxyglucose positron emission tomography combined with computed tomography, indicating a high risk of recurrence. Molecular markers, including vimentin, napsin A, phosphorylated c-Met, cytoplasmic maspin, Notch-1, MUC1, and tumoral CD10, may have higher expression in MPPAC than other subtypes; conversely, markers such as MUC4 and surfactant apoprotein A have lower expression in MPPAC. MPPAC with EGFR mutations can benefit from treatment with EGFR tyrosine kinase inhibitors. Furthermore, a complete lobectomy may be more suitable than limited resection for MPPAC because of the low sensitivity of intraoperative frozen sections and the high risk of lymph node metastasis. MPA benefits more from adjuvant chemotherapy than do other histological subtypes, whereas MPA does not benefit from adjuvant radiotherapy. Of note, MPP is associated with poor prognosis in early-stage lung adenocarcinoma, but the prognostic value of MPP is controversial in advanced-stage lung adenocarcinoma.
\end{abstract}

Keywords: lung adenocarcinoma, micropapillary, clinical impacts

\section{Introduction}

Lung cancer is universally acknowledged to be a lethal disease. ${ }^{1}$ In the USA, lung and bronchus cancer is predicted to be the second most common cancer in both men and women in 2015 as well as the leading cause of cancer death. ${ }^{2}$ Adenocarcinoma (ADC) is the most common histological subtype of lung cancer in most countries, accounting for almost half of lung cancers. ${ }^{3}$ Because of the remarkable heterogeneity of clinical manifestations, radiology, pathology, and molecular features among $\mathrm{ADC}$, there is a desperate need to devise a more detailed classification and reach a universally accepted criterion of ADC reclassification. Therefore, the international multidisciplinary classification of lung ADC has emerged at the right moment, sponsored by the International Association for the Study of Lung Cancer, American Thoracic Society, and European Respiratory Society. In the new ADC classification, some new terminologies and diagnostic criteria have been proposed. The term "predominant" is applied to describe invasive ADC, which is defined by assessment of histological patterns semiquantitatively in 5\% increments on resection specimens. ${ }^{4}$ The new classification also recommends that pathologists list every present subtype 
and the percentage of the subtype in the diagnostic reports. ${ }^{4,5}$ Additionally, micropapillary has been added as a new histological subtype. Micropapillary-predominant adenocarcinoma (MPA) is evaluated as a high-grade subtype with a poor prognosis. ${ }^{4}$ The micropapillary pattern (MPP) was first described in lung cancer by Silver and Askin in $1997 .{ }^{6}$ Then, in 2002, Amin et al observed that the MPP exists in some lung ADC and is apt to metastasize. ${ }^{7}$ In the 2004 WHO classification, lung adenocarcinoma with a micropapillary pattern (MPPAC) was identified on the basis of its aggressive biological behavior, but it was not considered to be a new histological subtype, owing to a lack of published evidence. ${ }^{8}$ However, in recent years, MPPAC has attracted increasing attention, especially regarding its association with poor prognosis, including the tendency toward recurrence and metastasis. ${ }^{9-11}$ In this review, we discuss the recent advances in the clinical manifestations, histopathologic characteristics and genetic mutations, prognosis and survival, and the therapeutic impacts of MPPAC.

\section{Clinical manifestations}

Patients often have a presentation similar to that of other subtypes of ADC. Moreover, some patients initially present with metastatic symptoms, especially enlarged lymph nodes. ${ }^{7}$ Multiple studies have shown that MPPAC is associated with the male sex ${ }^{12,13}$ and nonsmoking status. ${ }^{10,14-17}$ However, in some investigations, the differences were not significant with regard to sex ${ }^{16,17}$ or smoking status. ${ }^{18}$ Compared with other histological patterns of ADC, MPP has higher rates of lymphatic invasion, ${ }^{9,19,20}$ visceral pleural invasion, ${ }^{10,17,21,22}$ and lymph node metastases, $7,9,16,17,22-25$ which influence the choice of the surgical method to some extent (Table 1). Zhang et al have reported a correlation of smoking $(P=0.008)$ between MPP-positive (MPP $\geq 1 \%$ ) and MPP-negative (MPP <1\%) groups in a sample with 886 ADCs consisting of $246 \mathrm{MPP}$-positive cases. The authors also found a correlation with lymph node metastasis $(P<0.001)$, pleural invasion $(P=0.031)$, and lymphatic invasion $(P<0.001)$ with frequencies of $39.6 \%$ vs $14.6 \%, 30.3 \%$ vs $23.6 \%$, and $73.1 \%$ vs $13.1 \%$, respectively, but the correlation with sex was not statistically significant $(P=0.350) .{ }^{16}$

\section{Preoperative diagnosis}

Imaging tests have an important role in preoperative diagnosis in clinical practice. Many studies have investigated the promising role of predicting histological patterns by using computed tomography (CT) scans. MPPAC is usually a solid nodule but may include slight nonsolid components. ${ }^{26}$ Hence,
Table I Clinical features of MPPAC compared with conventional ADC without a MPP

\begin{tabular}{|c|c|c|}
\hline Variables & MPPAC & Conventional ADC \\
\hline$\overline{S e x}$ & Male & $\mathrm{N}^{\mathrm{a}}$ \\
\hline Smoking status & Nonsmoker & $\mathrm{N}$ \\
\hline Lymphatic invasion & High & Low \\
\hline Visceral pleural invasion & High & Low \\
\hline Lymph node metastases & High & Low \\
\hline \multicolumn{3}{|l|}{ CT findings } \\
\hline Nodule appearance & Part-solid & $\mathrm{N}$ \\
\hline Lobulation & Predominant & $\mathrm{N}$ \\
\hline Location in tumor & Peripheral & $\mathrm{N}$ \\
\hline Tumor size & Big & Small \\
\hline \multicolumn{3}{|l|}{ Biomarkers } \\
\hline Vimentin & High & Low \\
\hline Napsin A & High & Low \\
\hline pc-Met & High & Low \\
\hline Cytoplasmic maspin & High & Low \\
\hline Notch-I & High & Low \\
\hline $\mathrm{MUCl}$ & High & Low \\
\hline Tumoral CDIO & High & Low \\
\hline MUC4 & Low & High \\
\hline Surfactant apoprotein A & Low & High \\
\hline EGFR mutations & High & Low \\
\hline$B R A F$ mutation & High & Low \\
\hline
\end{tabular}

Note: a $\mathrm{N}$ indicates that there is no apparent feature or variability.

Abbreviations: MPPAC, lung adenocarcinoma with a micropapillary pattern; ADC, adenocarcinoma; MPP, micropapillary pattern; $\mathrm{SUV}_{\text {max }}$, maximum standardized uptake value; CT, computed tomography; pc-Met, phospho-c-Met.

Pass et al have proposed that surgeons should not choose limited resection (LR) on the basis of only the CT appearance of a solitary solid or partly solid nodule because the nodule may contain some MPP, which is a predictive factor of aggressive biological behavior. ${ }^{27}$ Austin et al have suggested that, in clinical practice, the size of the solid component of part-solid lung ADC may be more significant than the total size including the nonsolid component, which may influence the evaluation of T status (tumor size in tumor node metastasis [TNM] classification) in the next edition of the TNM classification system. ${ }^{26}$ Many studies have also found that using the size of the invasive component is more suitable than using the total size to evaluate T status. ${ }^{4,5,20,28}$ However, more studies are required to clarify the impact on the evaluation of $\mathrm{T}$ status. Furthermore, predominantly lobulated ADC may predict the presentation of MPP. ${ }^{29}$ The characteristics of CT such as tumor shape, sphericity, location, tumor disappearance ratio, and attenuation are not apparently different between MPPAC and other ADC, although these parameters may be related to malignant biological behaviors (Table 1). ${ }^{25,29-31}$

Fluorodeoxyglucose positron emission tomography combined with computed tomography (PET/CT) has gradually become routine for evaluating lung cancer staging. ${ }^{32}$ 
The maximum standardized uptake value $\left(\mathrm{SUV}_{\max }\right)$ in fluorodeoxyglucose PET/CT, as the main value of measure, describes a semiquantitative value of glucose uptake in organic lesions. Nakamura et al have reported an $\mathrm{SUV}_{\text {max }}$ of $5.78 \pm 3.40$ for MPA, representing the highest value among subtypes (Table 1). They have also shown that among the subgroups divided by histological classification (low, intermediate, and high grade), the higher the $\mathrm{SUV}_{\max }$, the greater the recurrent risk. ${ }^{33}$ In other words, MPP has a tendency toward recurrence. Yeh et al have made a further observation predicting occult lymph node metastasis in clinically mediastinal node-negative lung ADC. They have reported an association between $\mathrm{SUV}_{\text {max }}$ in PET/CT and the risk of $\mathrm{pN} 2$ disease in univariate analysis but not in multivariate analysis. The presence of MPP was significantly related to the risk of $\mathrm{pN} 2$ (pathologic lymph node status in TNM classification) disease in both univariate analysis and multivariate analysis. The result is also consistent with the tendency of MPP to metastasize to lymph nodes and suggests that MPP has a higher $\mathrm{SUV}_{\text {max }}$ than other histological patterns. ${ }^{34}$ This speculation has been further validated by several investigations. ${ }^{35-37}$

Apart from imaging diagnosis before surgery, pathologic diagnosis on the basis of small biopsies and cytology is of great importance, especially for advanced-stage lung ADC. However, pathologic diagnosis cannot adequately identify MPP from other ADCs. Rudomina et al have performed a retrospective analysis of 46 MPPACs with cytologic specimens (45 fine-needle aspirations of the lung and one of a pleural mass) and 33 ADCs without MPP for comparison. They found no significant difference between the two groups regarding the distribution of micropapillary tufts in the cytological materials and no relation with the percentage of MPP and the presence of micropapillary tufts. ${ }^{38}$ The discordance

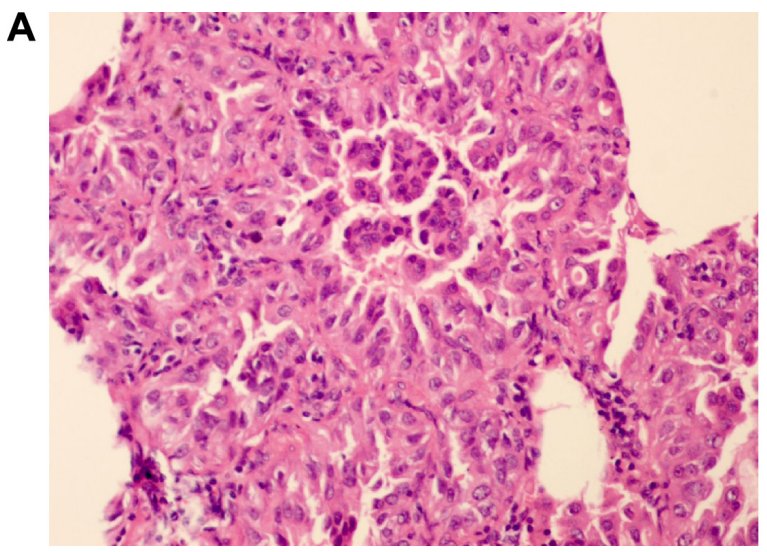

between resection samples and cytological specimens has also been reported by other studies..$^{31,39}$

\section{Histopathologic characteristics}

MPP is often discovered in the peripheral area of a nodule or mass. ${ }^{40,41}$ Histologically, MPP has been characterized by papillary tufts with no fibrovascular cores (Figure 1A and B), which is distinct from general tumors in which the vascularity is integral and important for the access to nourishment. ${ }^{4,7}$ To date, the mechanism of nourishment of MPP is uncertain, and these tumor cells may acquire nourishment from surrounding fluids in the alveolar spaces. ${ }^{42}$ Amin et al have classified MPP into two types: the classical type, in which micropapillary tufts float in the alveolar spaces or cluster in connective tissue spaces, and the variant type, in which micropapillary tufts float within cystic spaces lined by tumor cells. ${ }^{7}$

The cells of MPP are generally small and cuboidal with minimal nuclear atypia, detaching and/or connecting to alveolar walls. ${ }^{4,8}$ Because of the positive staining of E-cadherin and $\beta$-catenin, the negative staining of laminin, and the loss of the basement membrane, tight adhesion is validated to be present in MPP cells, whereas cell-matrix contact and cell polarity are absent. ${ }^{42}$ Kamiya et al have suggested that the disordered structure may contribute to the characteristics of metastasis. ${ }^{42}$

In primary tumors, almost all ADCs are a mixture of several histological subtypes, and the percentage of MPP varies. ${ }^{7,9,16,20,24,38,41,43-45}$ MPP is mainly observed at the periphery rather than in the center of the primary tumor, ${ }^{7,8}$ and the presentation of MPP in metastases is remarkably higher than in the primary tumors. ${ }^{4,7,8}$ Moreover, the concordance of histological subtype is $100 \%$ between the primary ADC and the metastatic tumor. ${ }^{46,47}$

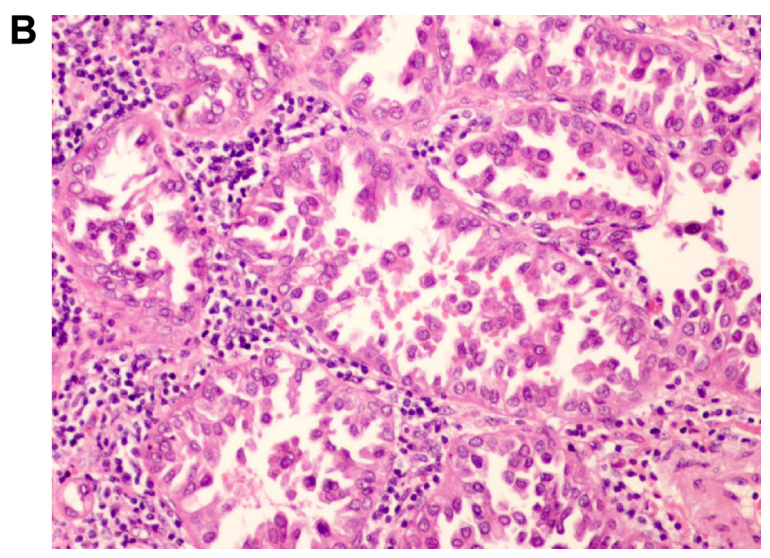

Figure I Pathological images show the morphology of a micropapillary pattern.

Notes: (A and B) Images showing hematoxylin-eosin staining at magnification $\times 200$. (A) Micropapillary tufts float in the alveolar space. (B) Lung adenocarcinoma cells cluster like micropapillary pattern and bulge into alveolar space. 


\section{Molecular features}

Recently, biomarker studies have become increasingly heated. With the development of biotechnology, molecular testing has gradually been put into clinical practice to identify the origin, histological type, and proper treatment strategies, especially for advanced lung cancer, which is diagnosed on the basis of small biopsies and cytology. Here, we introduce some special markers for MPP (Table 1).

\section{Vimentin}

Vimentin is a type-III intermediate filament. Vimentin intermediate filaments play an important role in the initiation and progression of lung cancer. ${ }^{48,49}$ In recent studies, vimentin has been found to have higher expression in MPPAC, and it is correlated with higher risks of vascular invasion and lymph node metastasis. ${ }^{50,51}$ In a sample of 101 MPPACs and 119 conventional ADCs of stages I-III, vimentin expression was detected by semiquantitative immunohistochemistry. Of the 101 MPPACs, vimentin expression scores of MPP and background non-MPP were 4.0 \pm 2.1 and 2.3 \pm 1.9 , respectively, with a $P$-value of $<0.0001$. Compared with 119 conventional ADCs, the vimentin expression score of MPP was significantly higher than that of well- and moderately differentiated conventional $\mathrm{ADC}(0.6 \pm 1.2$ and $1.9 \pm 1.7$, respectively), instead of that of poorly differentiated conventional ADC with the score of 3.8 \pm 2.7 . Moreover, vimentin expression scores in 101 MPPACs with vascular invasion and lymph node metastasis were $4.2 \pm 2.1$ and $4.4 \pm 1.9$, respectively, and were statistically higher than those without vascular invasion or node metastasis $(2.8 \pm 2.0$ and $3.3 \pm 2.3$, respectively), with $P=0.0196$ and $P=0.0122$, respectively. High vimentin expression in MPP was an independent adverse prognostic factor for both overall survival (OS) and disease-free survival (DFS) (hazards ratio $[\mathrm{HR}]=1.71,95 \%$ confidence interval $[\mathrm{CI}]=1.00-2.99, P=0.047) .{ }^{51}$

\section{Napsin A}

Napsin A is an aspartic proteinase and a marker for identifying primary lung ADC, which is more sensitive and specific than TTF-1. ${ }^{52}$ Warth et al have demonstrated that MPA has the highest frequency of napsin A expression compared with that in other predominant histological subtypes $(P<0.001) .^{53}$

\section{Phosphorylated c-Met}

The mesenchymal-epidermal transition (c-Met) protein and its ligand, hepatocyte growth factor, make a great influence on the prognosis and targeted therapy in non-small-cell lung cancer (NSCLC).${ }^{54}$ Koga et al have investigated a cohort of pT1-size lung ADC and have found that c-Met immunoreactivity was irrelevant to the presence of MPP but phospho-c-Met (pc-Met) was significantly more highly expressed in the MPP-positive (the percentage of MPP $\geq 10 \%$ ) group compared with the MPP-negative $(<10 \%)$ group, with a frequency of 0.293 and 0.100 , respectively $(P=0.01)$. Highly expressed pcMet in the MPP-positive group was statistically related to lymphatic involvement ( $P=0.0001), 82 \%$ vs $18 \%$, respectively. Only in p-stage IA ADC, highly expressed pc-Met was statistically adversely associated with 5-year survival compared with weakly expressed pc-Met, with a percentage of $51.3 \%$ and $79.4 \%(P=0.0313) .{ }^{55}$ Lee et al have declared a similar conclusion. ${ }^{36}$ Therefore, the phosphorylation of c-Met is related to MPP and probably contributes to the aggressive biological behavior of MPP. Therefore, crizotinib, as a c-Met inhibitor, may provide another option for MPPAC treatment.

\section{MUCI, MUC4, and surfactant apoprotein A}

Previous studies have indicated that high expression of $\mathrm{MUC1}^{56-58}$ and low expression of MUC4 $4^{59,60}$ and surfactant apoprotein $\mathrm{A}^{61}$ are related to an adverse prognosis in lung cancer. Tsutsumida et al have found higher expression of MUC1 and lower expression of MUC4 in the MPPpositive group (MPP $\geq 1 \%$ ) than the MPP-negative group. Additionally, a reduced expression of surfactant apoprotein A has been reported to be an unfavorable prognostic factor in MPPAC in small-sized ADC. ${ }^{62}$ Ohe et al confirmed the unfavorable prognostic value of surfactant apoprotein $A$ in MPPAC again. ${ }^{63}$

Apart from the aforementioned reports, there are other biomarkers associated with MPP that have been reported, such as higher expression of cytoplasmic maspin, ${ }^{64}$ Notch- $1,{ }^{65}$ and tumoral CD10. ${ }^{66}$ However, there is a lack of additional literature to validate the correlations between these biomarkers and the presence of MPP, so we will not clarify them in detail here. MPPAC also has molecular markers specifically related to ADC, for example, TTF-1.

\section{Genetic mutations}

Recently, gene detection has gradually become conventional for advanced lung cancer, ${ }^{28}$ and targeted drugs may be more efficient than traditional chemotherapy. EGFR mutations are one of the most frequent mutations detected and have been confirmed as predictors of response to tyrosine kinase inhibitors (TKIs), which are the first-line therapy 
in advanced-stage lung ADC with mutated $E G F R .{ }^{4} \mathrm{MPA}$ has a higher frequency of $E G F R$ mutations compared with other histological subtypes (Table 1)..$^{53,67-69}$ Warth et al have investigated $416 \mathrm{ADCs}$, and have found that, of $25 \mathrm{MPAs}$, $32 \%$ had EGFR mutations, which was higher than the $23.3 \%$ of lepidic-predominant ADC, $18.8 \%$ of acinar-predominant ADC, $9.4 \%$ of solid-predominant ADC, and $14.3 \%$ of papillary-predominant ADC. Therefore, it is crucial to detect the EGFR genetic status for MPA because it impacts initial therapy. However, because of ethnic differences and other factors, some controversial correlations between EGFR mutations and predominant histological subtypes have also been demonstrated. ${ }^{28,45,69-72}$ The frequency of EGFR mutations varies from $25 \%$ to $84.6 \%$ in MPPAC in variable cohorts and has been found to be higher in an Asian cohort. ${ }^{40,46,53,67,69,70,73-75}$ It is noteworthy that there is a controversy as to whether the frequency of EGFR mutations is associated with the percentage of MPP in the entire tumor. ${ }^{43,76}$ Regarding survival analysis, patients with MPP harboring EGFR mutations have been reported to have better survival when they received a TKI treatment compared with those with either no treatment ${ }^{76}$ or conventional platinum-based chemotherapy. ${ }^{13}$ Therefore, the application of $E G F R$ TKIs to $E G F R$-mutated patients with MPPAC may benefit in controlling the disease.

$B R A F$ is a downstream molecule in the EGFR signaling pathway, and its mutations play a role in resisting the function of EGFR TKIs. Some studies have shown that $B R A F$ mutations are more frequent in MPA than in other subtypes (Table 1). ${ }^{53,77}$ Warth et al have reported that $B R A F$ mutations were mainly present in MPA with a frequency of $8 \%$, higher than $0 \%$ of lepidic-predominant ADC, $4 \%$ of acinar-predominant ADC, $5.4 \%$ of solid-predominant ADC, and $0 \%$ of papillary-predominant ADC. To our knowledge, there is not a difference between MPA and other predominant histological subtypes with regard to $K R A S$ mutations or $A L K$ rearrangement. ${ }^{45,53,71,78}$

\section{Prognosis and survival}

An MPP has been reported in various cancers, such as breast ${ }^{79}$ thyroid,${ }^{80}$ bladder ${ }^{81}$ ovarian, ${ }^{82}$ renal, ${ }^{83}$ salivary,${ }^{84}$ colorectal, ${ }^{85,86}$ and lung cancers. For some of these tumors, the presentation of MPP has prognostic significance. The prognostic significance of MPP in lung ADC has also gradually become apparent. ${ }^{22,24,73,87}$ Warth et al have performed a study in 487 ADCs with surgery (stages I-IV) and have found that the predominant histological pattern has a statistically significant effect on survival. MPA had the poorest outcome compared with the other histological patterns with
OS of 44.9 \pm 6.3 months, disease-specific survival (DSS) of $50.4 \pm 6.7$ months, and DFS of $33.8 \pm 6.1$ months. ${ }^{20}$ Although the novel classification of ADC has defined MPA in 5\% increments, some studies indicated that even a minimal amount of MPP $(<5 \%$ increments) was associated with poor prognosis. ${ }^{16,62,75}$ Lee et al have classified 525 ADCs into three groups based on the percentage of MPP: 1$) \geq 5 \%$ of MPP ( $\mathrm{n}=114), 2)<5 \%$ (but $\geq 1 \%$ ) of MPP ( $\mathrm{n}=115)$, and 3 ) absent $(<1 \%)$ MPP ( $n=296)$. They found that OS was significantly better in the group with absent MPP compared with the other two groups, whereas the difference in OS was not significant between the $\geq 5 \%$ group and $<5 \%$ group. ${ }^{43}$ Until now, in terms of the influence of MPP on the prognosis of lung ADC, there has not been a well-defined criterion of the percentage of MPP or a consensus on whether MPP is predominant in the total tumor matters in prognosis. Zhang et al have demonstrated that MPA ( $\geq 5 \%$ of MPP) had a statistically worse recurrence-free survival compared with nonmicropapillarypredominant $\mathrm{ADC}$ with $\geq 5 \%$ MPP in stage I patients, whereas a similar correlation was not present in stage II-III patients. ${ }^{76}$ Moreover, there has been a fierce controversy about whether the percentage of MPP is proportionate to the poor prognosis. Kamiya et al have divided 383 cases into four groups according to the proportion of MPP: none ( $0 \%$ of the tumor), focal $(<10 \%)$, moderate $(<50 \%)$, and extensive $(\geq 50 \%)$. They observed from the survival curves that the prognosis was worse with the increase in proportion of MPP in tumors, and both DFS and OS for each of the latter three groups were worse than those for the group with no MPP. Comparisons among the latter three groups were absent, so whether statistical significance existed is uncertain. ${ }^{42}$ Zhang et al have divided 886 cases into four groups according to the extent of MPP in lung ADC, namely $<1 \%$, $1-5 \%, 5-50 \%$, and $\geq 50 \%$. They have reported a conclusion similar to that made by Kamiya et al. ${ }^{16}$ In contrast, Sumiyoshi et al have revealed that the mean percentages of MPP in the recurrence and nonrecurrence groups were $20.4 \%$ and $18.3 \%$, respectively, with no significance $(P=0.996) .{ }^{13}$ These studies further indicate the importance of both the identification of MPP and the determination of the percentage in pathological reports. More investigations are needed to resolve the former two problems in the future.

MPP has been validated as an unfavorable prognostic marker in early-stage lung ADC regardless of cohorts. . $^{13,47,88-94}$ However, the role of MPP, with regard to prognosis, is uncertain in advanced-stage lung ADC. Zhang et al have reported that MPA ( $\geq 5 \%$ of MPP) had statistically worse recurrencefree survival compared with nonmicropapillary-predominant 
ADC with $\geq 5 \%$ of MPP in stage I patients, whereas they did not find a similar correlation in stage II-III patients. ${ }^{76}$ Campos-Parra et al have claimed that high-grade ADC (micropapillary-, papillary-, and solid-predominant) is associated with better survival compared with intermediategrade ADC (lepidic- and acinar-predominant) in advanced ADC (stages IIIB and IV), and the median progression-free survival (PFS) and OS were 6.4 vs 5.5 months $(P=0.009)$ and 25 vs 16.8 months $(P=0.023)$, respectively. For this result, they considered that a better response to chemotherapy probably contributed to this phenomenon. ${ }^{95}$ Subsequently, Clay et al have also shown that MPP is not a predictor of unfavorable survival in stages III-IV.${ }^{74}$ In contrast, Cakir et al have indicated that the presentation of MPP is a predictor of unfavorable outcome in not only early-stage ADC but also late-stage $\mathrm{ADC}{ }^{18}$

\section{Therapeutic impacts}

\section{Influences on surgical treatment}

As described earlier, MPPAC usually presents as a solitary nodule or mass. The current gold standard operation of early-stage lung cancer is lobectomy (LO) with hilar and mediastinal lymph node dissection. ${ }^{96,97}$ With the development of imaging technology and the widespread use of CT screening, there has been a marked increase in the detection rate of small early-stage ADC. Herein, LR is used in place of LO in peripheral early-stage ADC because of its comparably curative effect and decreased damage to lung function. ${ }^{98,99}$ However, the prognostic utility of LR is uncertain. Hung et al have indicated that the micropapillary-/solid-predominant pattern is a marker of adverse prognosis $(P=0.003)$ in patients after complete resection of lung ADC. ${ }^{11}$ Therefore, considering the tendency toward recurrence and metastasis of MPP, a deliberate choice must be made in peripheral early-stage MPPAC. Nitadori et al have reported that, in 734 patients undergoing LR or LO for small ( $\leq 2 \mathrm{~cm}$ ) lung ADCs, in the LR group, $\geq 5 \% \mathrm{MPP}$ had a higher risk of recurrence than patients with $<5 \%$ MPP $(P<0.001)$. The 5-year cumulative incidences of recurrence were $34.2 \%$ and $12.4 \%$, respectively, and most recurrences were local recurrence; in the LR group, when the surgical margin was $<1 \mathrm{~cm}$, there was a similar outcome in terms of local recurrence ( $P=0.007$ ), but neither the LO group nor patients with a surgical margin of $\geq 1 \mathrm{~cm}$ showed statistically significant recurrence. ${ }^{100}$ In patients with $\mathrm{LR}, \geq 5 \%$ MPP is an independent predictor of recurrence. Subsequently, Bao et al have revealed that MPA is significantly more likely to present with pathological lymph node metastases in patients with clinical T1aN0 NSCLC. ${ }^{23}$ Kadota et al have also demonstrated the adverse predictive value of tumors that have spread through air spaces regarding recurrence after LR for small stage I ADC. ${ }^{101}$ Because of the tendency to recur, Ye et al have suggested that the intraoperative diagnosis of histological subtype should be made to determine whether systematic lymph nodes need dissection in clinical stage IA ADCs. ${ }^{19}$ Therefore, considering the underestimated lymph node status before surgery, a complete LO may be more suitable for MPPAC rather than an LR, and surgeons need to examine lymph nodes carefully during surgery.

In terms of intraoperative diagnosis, there is a lack of effective methods for diagnosing the presence of MPP during an operation. Intraoperative frozen sections are the primary method of diagnosis, which has been reported to have poor sensitivity for MPP. Yeh et al have assessed frozen sections from 361 ADCs in stage I with a tumor size $\leq 3 \mathrm{~cm}$, including 24 MPAs, and compared their concordance with permanent sections. The sensitivities of the frozen sections for acinar, lepidic, papillary, solid, and MPPs were 90\%, 75\%, 70\%, $69 \%$, and $37 \%$, respectively. ${ }^{102}$ Trejo et al have also reported a lower consistency between intraoperative frozen sections and permanent sections in MPA compared with other histological patterns in stage I lung ADC. ${ }^{103}$ Therefore, at present, permanent sections, as a postoperative diagnosis, are the only reliable method to identify the presence of MPP, rather than preoperative imaging, small biopsies, and cytology or frozen sections during an operation. ${ }^{31,104}$

\section{Influences on medical treatment}

Tumor recurrence after resection is still the primary reason leading to therapeutic failure. Chemotherapy, aside from surgery, is an indispensable part of the treatment of lung cancer, especially adjuvant chemotherapy for stage II-IIIA NSCLC, ${ }^{105}$ but to date, the role of adjuvant chemotherapy in stage IB NSCLC has been controversial. ${ }^{106} \mathrm{Xu}$ et al have studied prognostic factors in patients with completely resected lung ADC in stage IB. They found that histologic subtypes had a prognostic value in this cohort, but MPP was only a prognostic factor for PFS in univariate analysis rather than multivariate analysis. ${ }^{107}$ Tsao et al have designed a study on the predictive value of predominant histological patterns in lung ADC in stages I-III regarding survival benefitting from adjuvant chemotherapy. They reclassified micropapillary- and solid-predominant ADC as one group and found that this group benefitted from chemotherapy in both DFS $(\mathrm{HR}=0.60,95 \% \mathrm{CI}=0.44-0.82, P=0.001)$ and OS $(\mathrm{HR}=0.71,95 \% \mathrm{CI}=0.51-0.99, P=0.04)$ compared with other predominant histological patterns in multivariable 
analyses. Additionally, they investigated the predictive value of histological subtypes for chemotherapy response among different stages but found no statistical significance, although micropapillary- and solid-predominant ADCs in stages II and III rather than stage I had a tendency to respond better to chemotherapy than did the other subtypes presented. ${ }^{108}$ This study may indicate that MPP is sensitive to chemotherapy. As with adjuvant radiotherapy, Hung et al have shown that MPA could not improve the prognosis of adjuvant radiotherapy for stages I-III. ${ }^{9}$ In terms of the selection of chemotherapy regimens, Campos-Parra et al have demonstrated that MPA responded better than intermediate-grade ADC including lepidic- and acinar-predominant ADC, as demonstrated by the response rate ( $36.9 \%$ vs $25.4 \%, P=0.034$, respectively) and PFS (6.4 vs 5.5 months, $P=0.009$, respectively) after platinum-based chemotherapy in stages IIIB-IV; however, they lacked comparisons among different chemotherapy regimens in the cohort of MPA. ${ }^{95}$

\section{Conclusion and future prospects}

In conclusion, according to published studies, MPP in lung $\mathrm{ADC}$ has presented special clinical impacts, in particular, its influence on survival. However, numerous problems remain unaddressed. First, the diagnosis of MPP, whether preoperation, intraoperation, or postoperation, encounter challenges, especially in advanced-stage ADC, accounting for a lack of resection samples. More studies of molecular biomarkers and radiological findings are needed to identify MPP from other subtypes to stratify the prognosis of MPPAC. Experienced pathologists as well as specific and sensitive detection methods are required. The prognostic value of MPP needs to be elucidated clearly, including the associations between prognosis and the percentage of MPP and the status of MPP in the entire tumor (predominant or not) and tumor node metastasis stage. The mechanism of its aggressive biological behavior requires further elucidation, and the therapeutic response of MPP poses a problem because it influences the choice of operation and the postoperative management. There is a lack of reliable evidence to clarify the necessity of adjuvant chemotherapy for early-stage MPPAC, especially stage IB MPPAC. Last, given that recent studies have mostly been conducted in small populations, large-sample studies are needed that can comprehensively reveal the clinical features of MPP so that decisions about clinical management can be made.

\section{Disclosure}

The authors report no conflicts of interest in this work.

\section{References}

1. Allemani C, Weir HK, Carreira H, et al. Global surveillance of cancer survival 1995-2009: analysis of individual data for 25,676,887 patients from 279 population-based registries in 67 countries (CONCORD-2). The Lancet. 2015;385(9972):977-1010.

2. Siegel RL, Miller KD, Jemal A. Cancer statistics, 2015. CA: A Cancer Journal for Clinicians. 2015;65(1):5-29.

3. Matsuda T, Machii R. Morphological distribution of lung cancer from Cancer Incidence in Five Continents Vol. X. Japanese Journal of Clinical Oncology. 2015;45(4):404.

4. Travis WD, Brambilla E, Noguchi M, et al. International Association for the Study of Lung Cancer/American Thoracic Society/European Respiratory Society international multidisciplinary classification of lung adenocarcinoma. Journal of Thoracic Oncology: Official Publication of the International Association for the Study of Lung Cancer. 2011;6(2):244-285.

5. Travis WD, Brambilla E, Noguchi M, et al. International Association for the Study of Lung Cancer/American Thoracic Society/European Respiratory Society: international multidisciplinary classification of lung adenocarcinoma: executive summary. Proceedings of the American Thoracic Society. 2011;8(5):381-385.

6. Silver SA, Askin FB. True papillary carcinoma of the lung: a distinct clinicopathologic entity. The American Journal of Surgical Pathology. 1997;21(1):43-51.

7. Amin MB, Tamboli P, Merchant SH, et al. Micropapillary component in lung adenocarcinoma: a distinctive histologic feature with possible prognostic significance. The American Journal of Surgical Pathology. 2002;26(3):358-364.

8. Travis WD, Brambilla E, Noguchi M, et al. Diagnosis of lung adenocarcinoma in resected specimens: implications of the 2011 International Association for the Study of Lung Cancer/American Thoracic Society/ European Respiratory Society classification. Archives of Pathology \& Laboratory Medicine. 2013;137(5):685-705.

9. Hung JJ, Yeh YC, Jeng WJ, et al. Predictive value of the International Association for the Study of Lung Cancer/American Thoracic Society/ European Respiratory Society classification of lung adenocarcinoma in tumor recurrence and patient survival. Journal of Clinical Oncology: Official Journal of the American Society of Clinical Oncology. 2014; 32(22):2357-2364.

10. Hung JJ, Jeng WJ, Chou TY, et al. Prognostic value of the new International Association for the Study of Lung Cancer/American Thoracic Society/European Respiratory Society lung adenocarcinoma classification on death and recurrence in completely resected stage I lung adenocarcinoma. Annals of Surgery. 2013;258(6): 1079-1086.

11. Hung J-J, Yeh Y-C, Jeng W-J, et al. Prognostic factors of survival after recurrence in patients with resected lung adenocarcinoma. Journal of Thoracic Oncology. 2015;10(9):1328-1336.

12. Cha MJ, Lee HY, Lee KS, et al. Micropapillary and solid subtypes of invasive lung adenocarcinoma: clinical predictors of histopathology and outcome. The Journal of Thoracic and Cardiovascular Surgery. 2014;147(3):921-928.e922.

13. Sumiyoshi S, Yoshizawa A, Sonobe M, et al. Pulmonary adenocarcinomas with micropapillary component significantly correlate with recurrence, but can be well controlled with EGFR tyrosine kinase inhibitors in the early stages. Lung Cancer (Amsterdam, Netherlands). 2013;81(1):53-59.

14. Morales-Oyarvide V, Mino-Kenudson M. High-grade lung adenocarcinomas with micropapillary and/or solid patterns: a review. Current Opinion in Pulmonary Medicine. 2014;20(4):317-323.

15. Jain D. Low papillary structures in lepidic lung adenocarcinoma: any relationship with micropapillary lung adenocarcinoma? Human Pathology. 2013;44(12):2867.

16. Zhang J, Liang Z, Gao J, Luo Y, Liu T. Pulmonary adenocarcinoma with a micropapillary pattern: a clinicopathological, immunophenotypic and molecular analysis. Histopathology. 2011;59(6): 1204-1214. 
17. Miyoshi T, Satoh Y, Okumura S, et al. Early-stage lung adenocarcinomas with a micropapillary pattern, a distinct pathologic marker for a significantly poor prognosis. The American Journal of Surgical Pathology. 2003;27(1):101-109.

18. Cakir E, Yilmaz A, Demirag F, et al. Prognostic significance of micropapillary pattern in lung adenocarcinoma and expression of apoptosisrelated markers: caspase-3, bcl-2, and p53. APMIS: Acta Pathologica, Microbiologica, et Immunologica Scandinavica. 2011;119(9):574-580.

19. Ye B, Cheng M, Li W, et al. Predictive factors for lymph node metastasis in clinical stage IA lung adenocarcinoma. The Annals of Thoracic Surgery. 2014;98(1):217-223.

20. Warth A, Muley T, Meister M, et al. The novel histologic International Association for the Study of Lung Cancer/American Thoracic Society/European Respiratory Society classification system of lung adenocarcinoma is a stage-independent predictor of survival. Journal of Clinical Oncology: Official Journal of the American Society of Clinical Oncology. 2012;30(13):1438-1446.

21. Hamasaki M, Kato F, Koga K, et al. Invasion of the inner and outer layers of the visceral pleura in pT1 size lung adenocarcinoma measuring $</=3 \mathrm{~cm}$ : correlation with malignant aggressiveness and prognosis. Virchows Archiv: An International Journal of Pathology. 2012;461(5): 513-519.

22. Makimoto Y, Nabeshima K, Iwasaki H, et al. Micropapillary pattern: a distinct pathological marker to subclassify tumours with a significantly poor prognosis within small peripheral lung adenocarcinoma $(</=20 \mathrm{~mm})$ with mixed bronchioloalveolar and invasive subtypes (Noguchi's type C tumours). Histopathology. 2005;46(6): 677-684.

23. Bao F, Yuan P, Yuan X, Lv X, Wang Z, Hu J. Predictive risk factors for lymph node metastasis in patients with small size non-small cell lung cancer. Journal of Thoracic Disease. 2014;6(12):1697-1703.

24. Russell PA, Wainer Z, Wright GM, Daniels M, Conron M, Williams RA. Does lung adenocarcinoma subtype predict patient survival?: a clinicopathologic study based on the new International Association for the Study of Lung Cancer/American Thoracic Society/European Respiratory Society international multidisciplinary lung adenocarcinoma classification. Journal of Thoracic Oncology: Official Publication of the International Association for the Study of Lung Cancer. 2011;6(9): 1496-1504.

25. Wang $\mathrm{L}$, Jiang $\mathrm{W}$, Zhan $\mathrm{C}$, et al. Lymph node metastasis in clinical stage IA peripheral lung cancer. Lung Cancer (Amsterdam, Netherlands). 2015; 90(1):41-46.

26. Austin JH, Garg K, Aberle D, et al. Radiologic implications of the 2011 classification of adenocarcinoma of the lung. Radiology. 2013;266(1):62-71.

27. Pass HI. Lung cancer, histologic stratification, and resection extent: something for surgeons to think about. Journal of the National Cancer Institute. 2013;105(16):1168-1169.

28. Travis WD, Brambilla E, Riely GJ. New pathologic classification of lung cancer: relevance for clinical practice and clinical trials. Journal of Clinical Oncology: Official Journal of the American Society of Clinical Oncology. 2013;31(8):992-1001.

29. Lederlin M, Puderbach M, Muley T, et al. Correlation of radioand histomorphological pattern of pulmonary adenocarcinoma. The European Respiratory Journal. 2013;41(4):943-951.

30. Lee HY, Lee SW, Lee KS, et al. Role of CT and PET imaging in predicting tumor recurrence and survival in patients with lung adenocarcinoma: a comparison with the International Association for the Study of Lung Cancer/American Thoracic Society/European Respiratory Society classification of lung adenocarcinoma. Journal of Thoracic Oncology: Official Publication of the International Association for the Study of Lung Cancer. Epub 2015 Oct 15.

31. Eguchi T, Kadota K, Park BJ, Travis WD, Jones DR, Adusumilli PS. The new IASLC-ATS-ERS lung adenocarcinoma classification: what the surgeon should know. Seminars in Thoracic and Cardiovascular Surgery. 2014;26(3):210-222.

32. Lewis P, Marsden P, Gee T, et al. Whole-body 18F-fluorodeoxyglucose positron emission tomography in preoperative evaluation of lung cancer. The Lancet. 1994;344(8932):1265-1266.
33. Nakamura H, Saji H, Shinmyo T, et al. Close association of IASLC/ ATS/ERS lung adenocarcinoma subtypes with glucose-uptake in positron emission tomography. Lung Cancer (Amsterdam, Netherlands). 2015;87(1):28-33.

34. Yeh YC, Kadota K, Nitadori JI, et al. International Association for the Study of Lung Cancer/American Thoracic Society/European Respiratory Society classification predicts occult lymph node metastasis in clinically mediastinal node-negative lung adenocarcinoma. European Journal of Cardio-thoracic Surgery: Official Journal of the European Association for Cardio-thoracic Surgery. Epub 2015 Sep 15.

35. Mimae T, Miyata Y, Mimura T, et al. Radiologic findings to predict lowgrade malignant tumour among clinical $\mathrm{T} 1 \mathrm{bN} 0$ lung adenocarcinomas: lessons from histological subtypes. Japanese Journal of Clinical Oncology. 2015;45(8):767-773.

36. Lee MC, Kadota K, Buitrago D, Jones DR, Adusumilli PS. Implementing the new IASLC/ATS/ERS classification of lung adenocarcinomas: results from international and Chinese cohorts. Journal of Thoracic Disease. 2014;6(Suppl 5):S568-S580.

37. Kadota K, Colovos C, Suzuki K, et al. FDG-PET SUVmax combined with IASLC/ATS/ERS histologic classification improves the prognostic stratification of patients with stage I lung adenocarcinoma. Annals of Surgical Oncology. 2012;19(11):3598-3605.

38. Rudomina DE, Lin O, Moreira AL. Cytologic diagnosis of pulmonary adenocarcinoma with micropapillary pattern: does it correlate with the histologic findings? Diagnostic Cytopathology. 2009;37(5): 333-339.

39. Rodriguez EF, Monaco SE, Dacic S. Cytologic subtyping of lung adenocarcinoma by using the proposed International Association for the Study of Lung Cancer/American Thoracic Society/European Respiratory Society (IASLC/ATS/ERS) adenocarcinoma classification. Cancer Cytopathology. 2013;121(11):629-637.

40. Chao L, Yi-Sheng H, Yu C, et al. Relevance of EGFR mutation with micropapillary pattern according to the novel IASLC/ATS/ ERS lung adenocarcinoma classification and correlation with prognosis in Chinese patients. Lung Cancer (Amsterdam, Netherlands). 2014;86(2):164-169.

41. Roh MS, Lee JI, Choi PJ, Hong YS. Relationship between micropapillary component and micrometastasis in the regional lymph nodes of patients with stage I lung adenocarcinoma. Histopathology. 2004;45(6): 580-586.

42. Kamiya K, Hayashi Y, Douguchi J, et al. Histopathological features and prognostic significance of the micropapillary pattern in lung adenocarcinoma. Modern Pathology: An Official Journal of the United States and Canadian Academy of Pathology, Inc. 2008;21(8):992-1001.

43. Lee G, Lee HY, Jeong JY, et al. Clinical impact of minimal micropapillary pattern in invasive lung adenocarcinoma: prognostic significance and survival outcomes. The American Journal of Surgical Pathology. 2015;39(5):660-666.

44. Lee HY, Jeong JY, Lee KS, et al. Solitary pulmonary nodular lung adenocarcinoma: correlation of histopathologic scoring and patient survival with imaging biomarkers. Radiology. 2012;264(3):884-893.

45. Rekhtman N, Ang DC, Riely GJ, Ladanyi M, Moreira AL. KRAS mutations are associated with solid growth pattern and tumor-infiltrating leukocytes in lung adenocarcinoma. Modern Pathology: An Official Journal of the United States and Canadian Academy of Pathology, Inc. 2013; 26(10):1307-1319.

46. Russell PA, Barnett SA, Walkiewicz M, et al. Correlation of mutation status and survival with predominant histologic subtype according to the new IASLC/ATS/ERS lung adenocarcinoma classification in stage III (N2) patients. Journal of Thoracic Oncology: Official Publication of the International Association for the Study of Lung Cancer. 2013;8(4): 461-468.

47. Sica G, Yoshizawa A, Sima CS, et al. A grading system of lung adenocarcinomas based on histologic pattern is predictive of disease recurrence in stage I tumors. The American Journal of Surgical Pathology. 2010; 34(8):1155-1162.

48. Steinert PM, Roop DR. Molecular and cellular biology of intermediate filaments. Annual Review of Biochemistry. 1988;57:593-625. 
49. Kidd ME, Shumaker DK, Ridge KM. The role of vimentin intermediate filaments in the progression of lung cancer. American Journal of Respiratory Cell and Molecular Biology. 2014;50(1):1-6.

50. Hirano H, Maeda H, Takeuchi Y, et al. Lymphatic invasion of micropapillary cancer cells is associated with a poor prognosis of pathological stage IA lung adenocarcinomas. Oncology Letters. 2014;8(3): 1107-1111.

51. Nakashima H, Jiang SX, Sato Y, et al. Prevalent and up-regulated vimentin expression in micropapillary components of lung adenocarcinomas and its adverse prognostic significance. Pathology International. 2015; 65(4):183-192.

52. Turner BM, Cagle PT, Sainz IM, Fukuoka J, Shen SS, Jagirdar J. Napsin A, a new marker for lung adenocarcinoma, is complementary and more sensitive and specific than thyroid transcription factor 1 in the differential diagnosis of primary pulmonary carcinoma: evaluation of 1,674 cases by tissue microarray. Archives of Pathology \& Laboratory Medicine. 2012; 136(2):163-171

53. Warth A, Penzel R, Lindenmaier H, et al. EGFR, KRAS, BRAF and ALK gene alterations in lung adenocarcinomas: patient outcome, interplay with morphology and immunophenotype. The European Respiratory Journal. 2014;43(3):872-883.

54. Landi L, Minuti G, D'Incecco A, Cappuzzo F. Targeting c-MET in the battle against advanced nonsmall-cell lung cancer. Current Opinion in Oncology. 2013;25(2):130-136.

55. Koga K, Hamasaki M, Kato F, et al. Association of c-Met phosphorylation with micropapillary pattern and small cluster invasion in pT1-size lung adenocarcinoma. Lung Cancer (Amsterdam, Netherlands). 2013; 82(3):413-419.

56. Schroeder JA, Al Masri A, Adriance MC, et al. MUC1 overexpression results in mammary gland tumorigenesis and prolonged alveolar differentiation. Oncogene. 2004;23(34):5739-5747.

57. Castorina A, Giunta S. Mucin 1 (MUC1) signalling contributes to increase the resistance to cell death in human bronchial epithelial cells exposed to nickel acetate. Biometals. 2014;27(6):1149-1158.

58. Liu X, Ren H, Wu Z. Detecting MUC-1 mRNA for diagnosing peripheral blood micro-metastasis in non-small cell lung cancer patients. Chinese Journal of Cancer. 2008;27(12):1267-1270.

59. Majhi PD, Lakshmanan I, Ponnusamy MP, et al. Pathobiological implications of MUC4 in non-small-cell lung cancer. Journal of Thoracic Oncology. 2013;8(4):398-407.

60. Kwon KY, Ro JY, Singhal N, et al. MUC4 expression in non-small cell lung carcinomas - relationship to tumor histology and patient survival. Archives of Pathology \& Laboratory Medicine. 2007;131(4): 593-598.

61. Zamecnik J, Kodet R. Value of thyroid transcription factor-1 and surfactant apoprotein A in the differential diagnosis of pulmonary carcinomas: a study of 109 cases. Virchows Archiv: An International Journal of Pathology. 2002;440(4):353-361.

62. Tsutsumida H, Nomoto M, Goto M, et al. A micropapillary pattern is predictive of a poor prognosis in lung adenocarcinoma, and reduced surfactant apoprotein A expression in the micropapillary pattern is an excellent indicator of a poor prognosis. Modern Pathology: An Official Journal of the United States and Canadian Academy of Pathology, Inc. 2007;20(6):638-647.

63. Ohe M, Yokose T, Sakuma Y, et al. Stromal micropapillary component as a novel unfavorable prognostic factor of lung adenocarcinoma. Diagnostic Pathology. 2012;7:3.

64. Takagi Y, Matsuoka Y, Shiomi T, et al. Cytoplasmic maspin expression is a predictor of poor prognosis in patients with lung adenocarcinoma measuring $<3 \mathrm{~cm}$. Histopathology. 2015;66(5):732-739.

65. Huang J, Song H, Liu B, Yu B, Wang R, Chen L. Expression of Notch-1 and its clinical significance in different histological subtypes of human lung adenocarcinoma. Journal of Experimental \& Clinical Cancer Research: CR. 2013;32:84.

66. Kadota K, Buitrago D, Lee M-C, et al. Tumoral CD10 expression correlates with high-grade histology and increases risk of recurrence in patients with stage I lung adenocarcinoma. Lung Cancer (Amsterdam, Netherlands). 2015;89(3):329-336.
67. Song Z, Zhu H, Guo Z, Wu W, Sun W, Zhang Y. Correlation of EGFR mutation and predominant histologic subtype according to the new lung adenocarcinoma classification in Chinese patients. Medical Oncology (Northwood, London, England). 2013;30(3):645.

68. Shim HS, Lee da H, Park EJ, Kim SH. Histopathologic characteristics of lung adenocarcinomas with epidermal growth factor receptor mutations in the International Association for the Study of Lung Cancer/ American Thoracic Society/European Respiratory Society lung adenocarcinoma classification. Archives of Pathology \& Laboratory Medicine. 2011;135(10):1329-1334

69. Li H, Pan Y, Li Y, et al. Frequency of well-identified oncogenic driver mutations in lung adenocarcinoma of smokers varies with histological subtypes and graduated smoking dose. Lung Cancer (Amsterdam, Netherlands). 2013;79(1):8-13.

70. Tsuta K, Kawago M, Inoue E, et al. The utility of the proposed IASLC/ ATS/ERS lung adenocarcinoma subtypes for disease prognosis and correlation of driver gene alterations. Lung Cancer (Amsterdam, Netherlands). 2013;81(3):371-376.

71. Yoshizawa A, Sumiyoshi S, Sonobe M, et al. Validation of the IASLC/ ATS/ERS lung adenocarcinoma classification for prognosis and association with EGFR and KRAS gene mutations: analysis of 440 Japanese patients. Journal of Thoracic Oncology: Official Publication of the International Association for the Study of Lung Cancer. 2013; 8(1):52-61.

72. Sousa V, Rodrigues C, Silva M, Alarcao AM, Carvalho L. Lung adenocarcinoma: sustained subtyping with immunohistochemistry and EGFR, HER2 and KRAS mutational status. Revista Portuguesa De Pneumologia. 2015;21(3):113-125.

73. Yanagawa N, Shiono S, Abiko M, Ogata SY, Sato T, Tamura G. The correlation of the International Association for the Study of Lung Cancer (IASLC)/American Thoracic Society (ATS)/European Respiratory Society (ERS) classification with prognosis and EGFR mutation in lung adenocarcinoma. The Annals of Thoracic Surgery. 2014;98(2):453-458.

74. Clay TD, Do H, Sundararajan V, et al. The clinical relevance of pathologic subtypes in metastatic lung adenocarcinoma. Journal of Thoracic Oncology: Official Publication of the International Association for the Study of Lung Cancer. 2014;9(5):654-663.

75. Truini A, Pereira PS, Cavazza A, et al. Classification of different patterns of pulmonary adenocarcinomas. Expert Review of Respiratory Medicine. 2015;9(5):571-586.

76. Zhang Y, Wang R, Cai D, et al. A comprehensive investigation of molecular features and prognosis of lung adenocarcinoma with micropapillary component. Journal of Thoracic Oncology: Official Publication of the International Association for the Study of Lung Cancer. 2014;9(12):1772-1778.

77. Marchetti A, Felicioni L, Malatesta S, et al. Clinical features and outcome of patients with non-small-cell lung cancer harboring BRAF mutations. Journal of Clinical Oncology: Official Journal of the American Society of Clinical Oncology. 2011;29(26):3574-3579.

78. Finberg KE, Sequist LV, Joshi VA, et al. Mucinous differentiation correlates with absence of EGFR mutation and presence of KRAS mutation in lung adenocarcinomas with bronchioloalveolar features. The Journal of Molecular Diagnostics: JMD. 2007;9(3): 320-326.

79. Natrajan R, Wilkerson PM, Marchio C, et al. Characterization of the genomic features and expressed fusion genes in micropapillary carcinomas of the breast. The Journal of Pathology. 2014;232(5): $553-565$.

80. Burman KD. Micropapillary thyroid cancer: should we aspirate all nodules regardless of size? The Journal of Clinical Endocrinology and Metabolism. 2006;91(6):2043-2046.

81. Spaliviero M, Dalbagni G, Bochner BH, et al. Clinical outcome of patients with $\mathrm{T} 1$ micropapillary urothelial carcinoma of the bladder. The Journal of Urology. 2014;192(3):702-707.

82. Park JY, Kim DY, Kim JH, et al. Micropapillary pattern in serous borderline ovarian tumors: does it matter? Gynecologic Oncology. 2011;123(3): 511-516. 
83. Holmang S, Thomsen J, Johansson SL. Micropapillary carcinoma of the renal pelvis and ureter. The Journal of Urology. 2006;175(2):463-466; discussion 466-467.

84. Nagao T, Gaffey TA, Visscher DW, et al. Invasive micropapillary salivary duct carcinoma: a distinct histologic variant with biologic significance. The American Journal of Surgical Pathology. 2004;28(3): 319-326.

85. Barresi V, Branca G, Vitarelli E, Tuccari G. Micropapillary pattern and poorly differentiated clusters represent the same biological phenomenon in colorectal cancer: a proposal for a change in terminology. American Journal of Clinical Pathology. 2014;142(3):375-383.

86. Lee HJ, Eom DW, Kang GH, et al. Colorectal micropapillary carcinomas are associated with poor prognosis and enriched in markers of stem cells. Modern Pathology: An Official Journal of the United States and Canadian Academy of Pathology, Inc. 2013;26(8):1123-1131.

87. Sanchez-Mora N, Presmanes MC, Monroy V, et al. Micropapillary lung adenocarcinoma: a distinctive histologic subtype with prognostic significance. Case series. Human Pathology. 2008;39(3):324-330.

88. Zhang Y, Sun Y, Xiang J, Zhang Y, Hu H, Chen H. A clinicopathologic prediction model for postoperative recurrence in stage Ia non-small cell lung cancer. The Journal of Thoracic and Cardiovascular Surgery. 2014;148(4):1193-1199.

89. Yang F, Chen K, Liao Y, et al. Risk factors of recurrence for resected T1aN0M0 invasive lung adenocarcinoma: a clinicopathologic study of 177 patients. World Journal of Surgical Oncology. 2014;12:285.

90. Sun Y, Yu X, Shi X, Hong W, Zhao J, Shi L. Correlation of survival and EGFR mutation with predominant histologic subtype according to the new lung adenocarcinoma classification in stage IB patients. World Journal of Surgical Oncology. 2014;12:148.

91. Zhang J, Wu J, Tan Q, Zhu L, Gao W. Why do pathological stage IA lung adenocarcinomas vary from prognosis?: a clinicopathologic study of 176 patients with pathological stage IA lung adenocarcinoma based on the IASLC/ATS/ERS classification. Journal of Thoracic Oncology: Official Publication of the International Association for the Study of Lung Cancer. 2013;8(9):1196-1202.

92. Yeh YC, Wu YC, Chen CY, Wang LS, Hsu WH, Chou TY. Stromal invasion and micropapillary pattern in 212 consecutive surgically resected stage I lung adenocarcinomas: histopathological categories for prognosis prediction. Journal of Clinical Pathology. 2012;65(10):910-918.

93. Tsubokawa N, Mimae T, Sasada S, et al. Negative prognostic influence of micropapillary pattern in stage IA lung adenocarcinoma. European Journal of Cardio-thoracic Surgery: Official Journal of the European Association for Cardio-thoracic Surgery. Epub 2015 Mar 11.

94. Xu CH, Wang W, Wei Y, et al. Prognostic value of the new International Association for the Study of Lung Cancer/American Thoracic Society/ European Respiratory Society classification in stage IB lung adenocarcinoma. European Journal of Surgical Oncology: The Journal of the European Society of Surgical Oncology and the British Association of Surgical Oncology. 2015;41(10):1430-1436.

95. Campos-Parra AD, Aviles A, Contreras-Reyes S, et al. Relevance of the novel IASLC/ATS/ERS classification of lung adenocarcinoma in advanced disease. The European Respiratory Journal. 2014;43(5): 1439-1447.
96. Ginsberg RJ, Rubinstein LV. Randomized trial of lobectomy versus limited resection for T1 N0 non-small cell lung cancer. Lung Cancer Study Group. The Annals of Thoracic Surgery. 1995;60(3):615-622; discussion 622-623.

97. Rusch VW, Hawes D, Decker PA, et al. Occult metastases in lymph nodes predict survival in resectable non-small-cell lung cancer: report of the ACOSOG Z0040 trial. Journal of Clinical Oncology: Official Journal of the American Society of Clinical Oncology. 2011;29(32):4313-4319.

98. Wisnivesky JP, Henschke CI, Swanson S, et al. Limited resection for the treatment of patients with stage IA lung cancer. Annals of Surgery. 2010;251(3):550-554.

99. Kates M, Swanson S, Wisnivesky JP. Survival following lobectomy and limited resection for the treatment of stage I non-small cell lung cancer $<=1 \mathrm{~cm}$ in size: a review of SEER data. Chest. 2011;139(3): 491-496.

100. Nitadori J, Bograd AJ, Kadota K, et al. Impact of micropapillary histologic subtype in selecting limited resection vs lobectomy for lung adenocarcinoma of $2 \mathrm{~cm}$ or smaller. Journal of the National Cancer Institute. 2013;105(16):1212-1220.

101. Kadota K, Nitadori JI, Sima CS, et al. Tumor spread through air spaces is an important pattern of invasion and impacts the frequency and location of recurrences following limited resection for small stage I lung adenocarcinomas. Journal of Thoracic Oncology: Official Publication of the International Association for the Study of Lung Cancer. 2015;10(5):806-814.

102. Yeh YC, Nitadori JI, Kadota K, et al. Using frozen section to identify histological patterns in stage I lung adenocarcinoma of $\leq 3 \mathrm{~cm}$ : accuracy and interobserver agreement. Histopathology. 2015;66(7):922-938.

103. Trejo Bittar HE, Incharoen P, Althouse AD, Dacic S. Accuracy of the IASLC/ATS/ERS histological subtyping of stage I lung adenocarcinoma on intraoperative frozen sections. Modern Pathology: An Official Journal of the United States and Canadian Academy of Pathology, Inc. 2015;28(8):1058-1063.

104. Lee MC, Buitrago DH, Kadota K, Jones DR, Adusumilli PS. Recent advances and clinical implications of the micropapillary histological subtype in lung adenocarcinomas. Lung Cancer Management. 2014; 3(3):245-253

105. Mascaux C, Shepherd FA. Adjuvant chemotherapy after pulmonary resection for lung cancer. Thoracic Surgery Clinics. 2013;23(3): 401-410.

106. Howington JA, Blum MG, Chang AC, Balekian AA, Murthy SC. Treatment of stage I and II non-small cell lung cancer: diagnosis and management of lung cancer, 3rd ed: American College of Chest Physicians evidence-based clinical practice guidelines. Chest. 2013; 143(5 Suppl):e278S-e313S.

107. Xu S, Xi J, Jiang W, Lu S, Wang Q. Solid component and tumor size correlate with prognosis of stage IB lung adenocarcinoma. The Annals of Thoracic Surgery. 2015;99(3):961-967.

108. Tsao MS, Marguet S, Le Teuff G, et al. Subtype classification of lung adenocarcinoma predicts benefit from adjuvant chemotherapy in patients undergoing complete resection. Journal of Clinical Oncology. 2015;33(30):3439-3446.
OncoTargets and Therapy

\section{Publish your work in this journal}

OncoTargets and Therapy is an international, peer-reviewed, open access journal focusing on the pathological basis of all cancers, potential targets for therapy and treatment protocols employed to improve the management of cancer patients. The journal also focuses on the impact of management programs and new therapeutic agents and protocols on

\section{Dovepress}

patient perspectives such as quality of life, adherence and satisfaction The manuscript management system is completely online and includes a very quick and fair peer-review system, which is all easy to use. Visit http://www.dovepress.com/testimonials.php to read real quotes from published authors. 